\title{
"Stay off the greens"
}

\section{R Newton, C C Cunningham}

\section{Encouraging consultation questions}

T here is great power in the spoken word and doctors should beware misunderstandings. The first author spent some time in general practice. On a home visit to a man in bed with pneumonia he was bidding farewell to the man's wife at the front door when she said, "He is still off the greens doctor". The first author did not understand what she meant. She explained that 20 years previously her husband had had a bout of diarrhoea and vomiting. The senior partner had advised that he should "stay off the greens"presumably for a short period. For the 20 years that followed not one green leaf had passed this man's lips. Arguably, the most powerful medical intervention is the spoken word, yet how we communicate with families is little studied.

The hospital clinic is the active point of contact between families, their children, and local paediatric services. From the family's point of view this is an essential interchange, often viewed with apprehension for many weeks as a result of worry over underlying disease or illness, real or perceived. For the doctor on many services the clinic is a stressful time commitment with a varying agenda: identifying (not missing) significant illness, assuring where appropriate, giving adequate supervision for trainees, and avoiding them too making mistakes. A typical scenario would be:

"the parents in front of you are in
tears, the last child no better,
trainee at the door with a
question, over-running 40 minutes
already, and waiting lists
growing"

In that interchange between families and doctors moments are precious. Despite best efforts it is easy to get things continuously wrong. The authors found that children with epilepsy attending a clinic continued to misunderstand the nature of their problem. ${ }^{1}$ Another dimension was opened up in a study of bereavement consultations. Often it was irrational and biologically implausible fears which forever would have remained unpredicted and undealt with by doctors that were worrying parents most. ${ }^{2}$

With so much at stake it is surprising that so little attention has traditionally

been given in training to consultation practice and communication skills. For example, research giving the news of disability was conducted 20 years ago, but only recently formulated as a practice guideline ${ }^{4}$ and adopted into the undergraduate curriculum. The reviewer of our paper wrote:

\begin{abstract}
"One would be optimistic that paediatricians are generally more receptive to parent's views and concerns. Paediatricians will generally act in partnership with parents and child as opposed to the more overpowering role of many of our colleagues who treat adults. Arguably the younger population with whom we deal do feel naturally more empowered anyway and more prepared to ask questions than perhaps the older generation."
\end{abstract}

This certainly is a popular notion among paediatricians, but our view of ourselves can lead to complacency. It was not until the first author was observed by the second author for the purposes of research into effective consultation content that he realised how often the questions parents wish to ask go unanswered, how poor children's knowledge of their illness can be (even when long term and serious), and how a child may be bypassed in the consultation proceedings.

\section{HOW MIGHT WE SET ABOUT IMPROVING THINGS?}

Research does exist to guide us in the communication process to make best use of the consultation time and to communicate effectively, and we present here a brief review. It is noteworthy that this research is largely adult based (the result of our complacency?), but there is much paediatricians can learn from this. For most young people a medical consultation is not a place where they can naturally assert themselves-unless given specific leave to do so.

To succeed, consultations between children, their families, and the doctor require that they provide relevant information to the clinic doctors and make their information needs known. This implies the need for active participation by families we see, yet studies frequently report that doctors driven by the time constraint tend to dominate the information exchange, and that many patients attending adult clinics are reluctant to take the initiative..$^{5-7}$ Recurring themes are reluctance and a lack of opportunity to ask questions, ${ }^{8}$ yet more accurate recall of information has been found with adult patients who ask more questions. $^{9}$

Patients' questions help the doctor to assess patient information needs (agenda) and their understanding of the information provided. Failure to elicit these, especially when there is a mismatch between patient and doctor agendas, often results in communication failure. ${ }^{10}$ There will be an increasing requirement in Common Law to meet standards of information delivery, with new standards, for example, on patient consent and drug information.

Despite the recognition of such factors and increased communication skills training for doctors, studies continue to report difficulties with patient question asking. Although largely adult based they nonetheless offer important information for paediatric practice. Young and colleagues $^{11}$ used a postal questionnaire with over 14000 young Australian women to elicit their views on general practitioner (GP) consultations. While they found an overall high satisfaction rate, a significant number wanted more opportunity to ask questions. Kavanagh and Broom $^{12}$ interviewed 29 women about their experiences of diagnosis of a cervical abnormality. They found most wanted to participate more in the decisions about their care but expressed difficulties in getting the information they required. They felt unable to ask questions in the consultation and to overcome "the inherent power structure of medical practice" and "time pressures within the consultation". In the context of pharmacist-patient communication, Schomer and Wiederholt ${ }^{13}$ examined 358 interactions from 12 community pharmacies. They concluded that patient question asking was an important cue to communication and that it was imperative to find ways to increase question asking and understand why some patients were reluctant to ask questions.

\section{INTERVENTIONS STUDIED} Preconsultation training

Interventions to facilitate patient behaviour have addressed such issues. Roter ${ }^{14}$ provided a 10 minute preconsultation, conducted by health educators with patients to help them identify and formulate a list of questions. Using a randomised design she found the intervention group asked more direct questions and were more likely to keep 
subsequent appointments. They were, however, less satisfied than the controls and both they and the doctors expressed more anger and anxiety with the consultation. She proposed a three factor model for question asking:

(1) Predisposing factors: for example, belief that it was acceptable to ask questions

(2) Enabling factors: for example, ability to formulate, articulate, and remember questions

(3) Reinforcing factors: for example, getting relevant answers. ${ }^{15}$

Greenfield and colleagues, ${ }^{16}$ with a similar approach, used a similar 20 minute intervention with hospital outpatients and reported fewer negative consequences. Although successful, this is an expensive method and is unlikely to be needed by all patients in all contexts. Thus it is not cost efficient.

\section{Preconsultation written material}

Other studies have attempted to provide patients with preconsultation written information. Robinson and Whitfield ${ }^{17}$ randomised patients into three groups, each given written information prior to the consultation. The controls were told about taking part in a project looking at patient-doctor communication. The second group had additional information encouraging them to ask questions, and the third additional information on strategies to formulate questions and check their understanding of what was said. The third group fared best, asked more questions, and recalled more information.

Recently, Butow and colleagues ${ }^{18}$ developed a question prompt sheet containing 11 potential questions for patients attending their first consultation with a medical oncologist. A total of 142 patients were randomised into those given the prompt sheet and those given a general booklet on services. The prompt sheet did not increase the overall number of questions asked, except for the area of prognosis. The authors suggest the doctor involved may have discouraged questions about prognosis as it is the most emotionally laden topic and possibly the most difficult for patients to address at the first consultation. They also found age, in or outpatient status, preferences for involvement and gender were predictive of both the number and duration of patients' questions, with younger women asking the most.

McCann and Wienman ${ }^{19}$ developed a leaflet, "Speak for Yourself", aimed at helping patients waiting to see one GP to identify their problem, write down their ideas about cause and treatment, and clearly state these during the consultation. The 59 in the intervention group asked more questions and had longer consultations than the 61 controls. Younger patients, and those from social classes 1 and 2 appeared to benefit more, but no differences were found in overall satisfaction.

Davison and Degner ${ }^{20}$ provided 60 newly diagnosed men with prostate cancer an information pack, and for 30 of them an additional prompt list of questions and an audiotape of the consultation. The latter had significantly higher levels of participation in the treatment decision and lower state anxiety levels at six weeks post-intervention.

Frederickson and Bull ${ }^{21}$ randomised 80 patients attending one GP into control and intervention groups. The intervention group received a folded single page A4 leaflet, "The Patient's Guide to Consultation", aimed at focusing their attention on why they were attending and what they hoped to get from the consultation. It also instructed them to state this early in the consultation and to ask questions during the consultation. All 40 intervention patients rated the leaflet as a good idea; several noted that writing down points to remember would be helpful, although some thought it might be frowned on. Patients' comments indicated that the leaflet had conveyed the message that the doctor was open to questions and welcomed discussion. The doctor, blind to who had received the leaflet, rated all consultations for quality of communication. There was a significant difference in favour of the intervention group. The authors note that the doctor involved was sympathetic to the ethos of the study, but had feared it would produce vast lists of problems and demands that he would not have the time to deal with. In the event this did not happen. In effect the consultations were more efficient.

\section{WHAT DO THESE STUDIES TEACH US?}

First, there is an assumption in many of these studies that patients need to be educated in "consultation skills", inferring these are lacking. Clearly many patients will have such skills but some may lack the assertiveness to present their agenda and ask questions. The studies partially support this suggestion as several note that the act of providing written information on specific conditions appears to act as an invitation by the doctor for the patient to become more actively engaged. The internet increasingly should offer us opportunities in this respect. For many patients this may be sufficient to have positive results.

Cunningham and Newton ${ }^{22}$ investigated this with parents attending two hospital based paediatric neurology clinics. A single sheet (fig 1), which merely invited parents to write down questions, was produced and evaluated with a range of consumers. The preferred sheet was then offered to parents attending one clinic over a period of 13 weeks. The other clinic acted as a comparison. Following the consultation parents completed a rating form and at the end of the period the doctors were interviewed. One third of the parents declined the sheet; several of these were seeking a diagnosis and stated they did not know what to ask until they were given the diagnosis. Of those using it, $88 \%$ liked it, $83 \%$ found it useful, and $71 \%$ wished to use it at future consultations.

Doctors reported that many issues, irrational fears, and misunderstandings emerged which they normally failed to identify (just as in the bereavement study ${ }^{2}$ ). Examples include the mother of a boy with a diplegia and periventricular leucomalacia (PVL). She had dropped him when he was 3 months old and was now worried it was she who had caused the brain injury (despite earlier information on the cause of PVL). In a second family the belief was that a cousin had cerebral palsy following and caused by a lumbar puncture. A lumbar puncture was planned in their own child as part of elective investigations. Neither family, for their own reasons had previously voiced their fears. The question sheet offered an opportunity to introduce the topic with less embarrassment and importantly an opportunity to resolve the issue. Doctors' pretrial fears of increased time and disruption from use of the question sheet did not materialise but instead, positive changes occurred in the nature of the consultations with more parents taking initiatives and control, particularly those on subsequent visits. No changes were observed in the comparison clinic.

There is some controversy over time and the intensity of the interaction in consultations. Roter ${ }^{14}$ found intensity (that is, the amount of information conveyed within a certain time) was positively associated with satisfaction, and Buller and Buller ${ }^{23}$ found that increased time spent talking was inversely related to patient satisfaction. The implication is that quality is more important than quantity and that this will arise when patients communicate their agenda clearly and doctors respond appropriately. Most of the evidence suggests that encouraging patients to ask questions does not inevitably increase consultation time, and where this becomes an expected part of the consultation process it can be more efficient and more satisfactory to both patients and doctors.

Despite methodological limitations, these studies generally indicate that giving patients preconsultative written information or formal requests to prepare questions for the consultation can 
most of us

come out after seeing the doctor

and say:

\section{"I forgot to ask......"}

PLEASE WRITE DOWN THE QUESTIONS THAT YOU WANT TO ASK AND GIVE THEM TO YOUR DOCTOR tick box if we may use these questions to help produce information booklets

this is confidential
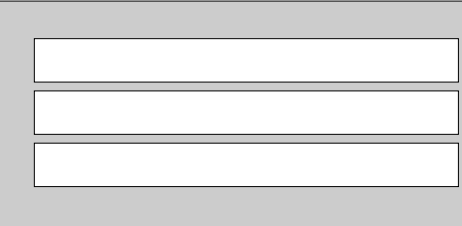

Figure 1 The author's question sheet (banner headline is highlighted in yellow).

result in more active participation and question asking and some positive outcomes for both doctors and patients. The studies also highlight the variability of usefulness related to patient variables such as age and gender, and the reason for the consultation and hence its content (diagnosis, prognosis, check up) and context (for example, first consultation with new doctor).

Thus each practitioner will need to consider the nature of their context and the patients they see. It would seem that creating an ethos for accurate information exchange and open, nonjudgemental communication, can be facilitated cheaply and quickly by offering some simple question sheet prior to the consultation. Depending on the patient and the condition, more complex written information may be required; for example, some indication of the questions that patients might need to consider after being given a potentially severe diagnosis. Finally, it would appear that this area can be explored with small scale, in-house, clinically oriented projects which are inexpensive yet may have considerable benefit. The advent of web based information technology will allow the more effective of these to be more accessible for the benefit of others.

Arch Dis Child 2003;88:278-280

\section{Authors' affiliations}

R Newton, Consultant Child and Adolescent Neurologist, Royal Manchester Children's Hospital, Pendlebury, Manchester M27 4HA UK

C C Cunningham, Visiting Professor in Applied Psychology, School of Health, Liverpool John Moores University, 79 Tithebarn Street, Liverpool L2 2ER, UK

Correspondence to: Dr R Newton, Consultan Child and Adolescent Neurologist, Royal Manchester Children's Hospital, Pendlebury, Manchester M27 4HA;

Richard.Newton@cmmc.nhs.uk

\section{REFERENCES}

1 Houston EC, Cunningham CC, Metcalfe E, et al. The information needs and understanding of 5-10 year old children with epilepsy, asthma, or diabetes. Seizure 2000;9:340-3.

2 Newton RW, Bergin B, Knowles D. Parents interviewed after their child's death. Arch Dis Child 1986;61:711-15.

3 Cunningham CC, Morgan PA, McGucken RB. Down's syndrome: is dissatisfaction with diagnosis inevitable? Dev Med Child Neurol 1984;26:33-9.

4 Lingam S, Newton RW. Giving the news of disability-paediatric practice guideline. London: Royal College of Paediatrics and Child Health, 1996.

5 Friedson E. Client control and medical practice. In: Jacob E, ed. Patients, physicians and illness. New York: Free Press, 1972.

6 Fletcher C. Listening and talking to patients I: the problem. BMV 1980;281:845-7.

7 Roter DL. Which facets of communication have strong effects on outcome-a meta-analysis. In: Stewart M, Roter DL, eds. Communicating with medical patients. London: Sage, 1989.

8 Byrne PS, Long BEL. Doctors talking to patients. London: HSMO, 1976.

9 Heszen-Klemens I, Lapinska E. Doctor-patient interaction, patients' health behaviour and effects of treatment. Soc Sci Med 1984;19:9-18.

10 Tuckett D, Boulton M, Olsen C. A new approach to the measurement of patients understanding of what they are told in medical consultations. J Health Soc Behav 1985;26:27-38

11 Young AF, Byles JE, Dobson AJ. Women's satisfaction with general practice consultations. Med J Aust 1998;168:386-9.

12 Kavanagh AM, Broom DH. Women's understanding of abnormal cervical smear test results: a qualitative interview study. $B M$ 1977;314:1388-91.

13 Schommer JC, Wiederholt JB. The association of prescription status, patient age, patient gender, and patient question asking behaviour with the content of

pharmacist-patient communication. Pharm Res 1997; 14:145-51.

14 Roter DL. Patient participation in the patient-provider interactions: the effects of patient question-asking on the quality of interactions, satisfaction and compliance. Health Educ Monogr 1977;5:281-312.

15 Roter DL. Patient question asking in physician-patient interaction. Health Psychol 1984;3:394-409.

16 Greenfield S, Kaplan S, Ware JE. Expanding patient involvement in care. Ann Int Med 1985; 102:520-8

17 Robinson EJ, Whiffield M. Improving the efficiency of patients' comprehension monitoring: a way of increasing patients' participation in general practice consultation. participation in general practice

18 Butow PN, Dunn SM, Tattersall MHN, et al. Patient participation in the cancer consultation: evaluation of a question prompt sheet. Ann Oncol 1994;5: 199-204.

19 McCann S, Weinman L. Empowering the patient in the consultation: a pilot project. Patient Educ Couns 1996;27:227-34.

20 Davidson BJ, Degner LF. Empowerment of men newly diagnosed with prostate cancer. Cancer Nurs 1997;20:187-96.

21 Frederikson LG, Bull PE. Evaluation of a patient education leaflet designed to improve communication in medical consultations. Patient Educ Couns 1995;25:51-7.

22 Cunningham CC, Newton RW. A question sheet to encourage written consultation questions. Qual Health Care 2000;9:42-6.

23 Buller MK, Buller DB. Physicians' communication style and patient satisfaction. J Health Soc Behav 1987;28:375-81. 